\title{
ON UNIFORM CONVERGENCE OF FOURIER SERIES
}

\author{
OTTO SZÁSZ
}

1. Introduction. In this section we collect some known concepts and simple facts, pertinent to our subject.

Given a sequence of real numbers $s_{n}, n \geqq 0$, consider for any $\lambda>1$

$$
\limsup _{n \rightarrow \infty} \max _{n<m \leqq \lambda n}\left(s_{m}-s_{n}\right)=u(\lambda) \leqq+\infty ;
$$

clearly $u(\lambda)$ decreases as $\lambda \downarrow 1$; if

$$
\lim _{\lambda \rightarrow 1} u(\lambda) \leqq 0,
$$

then the sequence $\left\{s_{n}\right\}$ is called slowly oscillating from above; similarly slow oscillation from below is defined by

$$
\lim _{\lambda \rightarrow 1} \liminf _{n \rightarrow \infty} \min _{n<m \leqq \lambda n}\left(s_{m}-s_{n}\right) \geqq 0 .
$$

If both (1.1) and (1.2) hold, that is if

$$
\lim _{\lambda \rightarrow 1} \limsup _{n \rightarrow \infty} \max _{n<m \leq \lambda n}\left|s_{m}-s_{n}\right|=0,
$$

then the sequence is called simply slowly oscillating. If $s_{n}=\sum_{0}^{n} a_{\nu}$ is the $n$th partial sum of a series $\sum_{0}^{\infty} a_{v}$, then (1.3) can be written as

$$
\lim _{\lambda \rightarrow 1} \limsup _{n \rightarrow \infty} \max _{n<m \leqq \lambda n}\left|\sum_{n+1}^{m} a_{\nu}\right|=0 .
$$

A more restricted class of series is defined by

$$
\lim _{\lambda \rightarrow 1} \limsup _{n \rightarrow \infty} \sum_{n<\nu \leqq \lambda n}\left|a_{\nu}\right|=0 .
$$

Special cases: If for some $p>0, n\left|a_{n}\right|<p$ for all $n$, then

$$
\sum_{n<\nu \leqq \lambda n}\left|a_{\nu}\right|<p \sum_{n}^{\lambda n} \frac{1}{\nu}=O(\log \lambda) .
$$

Hence (1.5) holds.

If only

$$
n a_{n}>-p \quad \text { for all } n,
$$

then

Presented to the Society, September 12, 1943; received by the editors January 19, 1944. 


$$
n\left(\left|a_{n}\right|-a_{n}\right)<2 p
$$

hence

$$
\lim _{\lambda \rightarrow 1} \limsup _{n \rightarrow \infty} \sum_{n}^{\lambda n}\left(\left|a_{\nu}\right|-a_{\nu}\right)=0 .
$$

This relation implies (1.2), but not necessarily (1.4). The following lemma is immediate:

LEMMA 1. Every convergent series satisfies (1.3); furthermore (1.3) and (1.6) imply (1.5).

A sequence of functions $s_{n}(t)$, defined at a point set $\varepsilon$ having $t=\tau$ for a limit point, is said to be uniformly convergent at $t=\tau$ if $\lim s_{n}\left(t_{n}\right)$ exists for any sequence $t_{n} \rightarrow \tau$. It is an immediate consequence of the definition that the limit of $s_{n}\left(t_{n}\right)$ is then unique.

If for each $n, s_{n}(t)$ is defined and continuous at $t=\tau$, then clearly a necessary condition for uniform convergence at $t=\tau$ is that $\lim _{n \rightarrow \infty} s_{n}(\tau)$ $=s$ exists.

We restrict ourselves to such sequences; then the following lemma holds:

LEMMA 2. The following two properties are equivalent:

(a) $s_{n}\left(t_{n}\right) \rightarrow s$ as $t_{n} \rightarrow \tau$;

(b) $s_{n}(\tau) \rightarrow s$, and $\left|s_{n}(\tau)-s_{n}(t)\right|<\epsilon$ for any $\epsilon>0$, and for $|\tau-t|<\delta(\epsilon)$, $n>n_{0}(\delta, \epsilon)=n_{0}(\epsilon)$.

Thus either (a) or (b) defines uniform convergence at $t=\tau$.

For the proof assume that (a) holds; if (b) would not hold, there would exist an $\epsilon=\epsilon_{0}$, so that $\lim \sup _{t_{n} \rightarrow \tau}\left|s_{n}(\tau)-s_{n}\left(t_{n}\right)\right|>\epsilon_{0}$. But this contradicts (a). Similarly if (b) holds, then (a) follows.

2. The cosine series. We now prove the following theorem.

Theorem 1. Suppose that the coefficients of the Fourier cosine series

$$
\phi(t) \sim a_{0} / 2+\sum_{1}^{\infty} a_{n} \cos n t
$$

satisfy the condition (1.6), and that $\phi(t)$ is continuous at $t=0$; then the series (2.1) is uniformly convergent at $t=0$.

Let

$$
s_{0}=\frac{a_{0}}{2}, \quad s_{n}(t)=\frac{a_{0}}{2}+\sum_{1}^{n} a_{\nu} \cos \nu t, \quad \sigma_{n}(t)=\frac{1}{n+1} \sum_{0}^{n} s_{\nu}(t) .
$$


By a theorem of Fejér $[1]^{1}$

$$
\sigma_{n}\left(t_{n}\right) \rightarrow \phi(0)
$$

as $t_{n} \rightarrow 0$;

in particular

$$
\sigma_{n}(0) \rightarrow \phi(0)
$$

as $n \rightarrow \infty$.

By a well known theorem of Tauberian type, (2.3) and (1.6) (or only (1.2)) imply that

$$
s_{n}(0) \rightarrow \phi(0) \text {. }
$$

By Lemma 1, (1.6) and (2.4) imply (1.5). We next employ the often used identity

$$
\begin{array}{r}
s_{n}-\sigma_{n+\nu}=\frac{n}{\nu+1}\left(\sigma_{n+\nu}-\sigma_{n-1}\right)-\frac{1}{\nu+1} \sum_{k=1}^{\nu}(\nu-k+1) c_{n+k}, \\
n \geqq 1, \nu \geqq 1,
\end{array}
$$

where $s_{n}, \sigma_{n}$ are the partial sums and arithmetical means respectively of the series $\sum c_{n}$. Thus

$$
\begin{aligned}
s_{n}(0)-s_{n}(t)- & \left\{\sigma_{n+\nu}(0)-\sigma_{n+\nu}(t)\right\} \\
= & \frac{n}{\nu+1}\left\{\sigma_{n+\nu}(0)-\sigma_{n+\nu}(t)-\left[\sigma_{n-1}(0)-\sigma_{n-1}(t)\right]\right\} \\
& -\frac{1}{\nu+1} \sum_{1}^{\nu}(\nu-k+1)[1-\cos (n+k) t] a_{n+k .} .
\end{aligned}
$$

By (2.2) and Lemma 2

$$
\left|\sigma_{n}(0)-\sigma_{n}(t)\right|<\epsilon \text { for }|t|<\delta(\epsilon) \text { and } n \geqq n_{0}(\epsilon) ;
$$

hence, from (2.6)

$$
\left|s_{n}(0)-s_{n}(t)\right|<\epsilon+\frac{2 n \epsilon}{\nu+1}+\frac{2}{\nu+1} \sum_{1}^{\nu}(\nu-k+1)\left|a_{n+k}\right|
$$

Write

$$
\begin{aligned}
<\epsilon+\frac{2 n \epsilon}{\nu+1}+2 \sum_{1}^{\nu}\left|a_{n+k}\right|, \\
|t|<\delta(\epsilon), n>n_{0}(\epsilon) .
\end{aligned}
$$

then

$$
\limsup _{n \rightarrow \infty} \sum_{n}^{\lambda n}\left|a_{\nu}\right|=\omega(\lambda)
$$

${ }^{1}$ Numbers in brackets refer to the literature listed at the end of the paper. 


$$
\sum_{n}^{\lambda n}\left|a_{\nu}\right|<\epsilon+\omega(\lambda) \quad \text { for } n>n_{1}(\epsilon, \lambda) \text {. }
$$

Given $\epsilon>0$, choose $\nu=\left[n \epsilon^{1 / 2}\right]$, and $\lambda=1+\epsilon^{1 / 2}$, then, from (2.7) and (2.8),

$$
\left|s_{n}(0)-s_{n}(t)\right|<\epsilon+2 \epsilon^{1 / 2}+2\left(\epsilon+\omega\left(1+\epsilon^{1 / 2}\right)\right) \quad \text { for } n>n_{2}(\epsilon),
$$

when $n_{2}$ is the larger of the two numbers $n_{0}, n_{1}$. The theorem now follows from (1.5) and Lemma 2.

The identity

$$
s_{n}(t)-\sigma_{n}(t)=\frac{1}{n+1} \sum_{1}^{n} \nu a_{\nu} \cos \nu t
$$

yields the corollary:

Corollary to Theorem 1. Under the assumptions of Theorem 1 $n^{-1} \sum_{1}^{n} \nu a_{\nu} \cos \nu t \rightarrow 0$ uniformly at $t=0$.

3. The sine series. In this case convergence at $t=0$ is trivial; we introduce two lemmas.

Lemma 3. Suppose that the coefficients of the Fourier sine series

$$
\psi(t) \sim \sum_{1}^{\infty} b_{n} \sin n t
$$

satisfy the condition (1.2) with $s_{n}=\sum_{1}^{n} b_{\nu}$, and that

$$
2 h^{-1} \int_{0}^{h} \psi(t) d t \rightarrow d
$$

then

$$
n^{-1} \sum_{1}^{n} \nu b_{\nu} \rightarrow \pi^{-1} d
$$

This is Lemma 6 of our paper [6].

LEMMA 4. If for a sequence $\left\{b_{n}\right\}$

exists, and if

$$
\lim n^{-1} \sum_{1}^{n} \nu b_{\nu}=l
$$

$$
\lim _{\lambda \downarrow 1} \limsup _{n \rightarrow \infty} \sum_{n}^{\lambda n}\left(\left|b_{\nu}\right|-b_{\nu}\right)=0
$$

then 


$$
\lim _{\lambda \downarrow 1} \limsup _{n \rightarrow \infty} \sum_{n}^{\lambda n}\left|b_{\nu}\right|=0 \text {. }
$$

Write

$$
\limsup _{n \rightarrow \infty} \sum_{n}^{\lambda n}\left(\left|b_{\nu}\right|-b_{\nu}\right)=\xi(\lambda), \quad \lambda>1,
$$

then by (3.2), $\xi(\lambda) \rightarrow 0$ as $\lambda \downarrow 1$. We have

hence

$$
\sum_{n}^{\lambda n} \nu\left(\left|b_{\nu}\right|-b_{\nu}\right) \leqq \lambda n \sum_{n}^{\lambda n}\left(\left|b_{\nu}\right|-b_{\nu}\right),
$$

Furthermore

$$
\lim \sup n^{-1} \sum_{n}^{\lambda n} \nu\left(\left|b_{\nu}\right|-b_{\nu}\right) \leqq \lambda \xi(\lambda)
$$

$$
n^{-1} \sum_{n}^{\lambda n} \nu b_{\nu}=\lambda(\lambda n)^{-1} \sum_{1}^{\lambda n} \nu b_{\nu}-n^{-1} \sum_{1}^{n-1} \nu b_{\nu} \rightarrow(\lambda-1) l,
$$

hence

But

hence

$$
\lim \sup n^{-1} \sum_{n}^{\lambda n} \nu\left|b_{\nu}\right| \leqq(\lambda-1) l+\lambda \xi(\lambda) .
$$

$$
\sum_{n}^{\lambda n}\left|b_{\nu}\right| \leqq n^{-1} \sum_{n}^{\lambda n} \nu\left|b_{\nu}\right|
$$

$$
\lim \sup \sum_{n}^{\lambda n}\left|b_{\nu}\right| \leqq(\lambda-1) l+\lambda \xi(\lambda) .
$$

Letting $\lambda \downarrow 1$, we get (3.3).

THeOREM 2. Suppose that the function $\psi(t)$ is continuous at $t=0$, that is $\psi(0)=0$, and that its Fourier coefficients satisfy (3.2). Then $\sum_{1}^{n} \nu b_{\nu}=o(n)$, and the series (3.1) is uniformly convergent at $t=0$.

We now write

$$
s_{n}(t)=\sum_{1}^{n} b_{\nu} \sin \nu t, \quad \sigma_{n}(t)=\frac{1}{n+1} \sum_{1}^{n} s_{\nu}(t) ;
$$

then by the theorem of Fejerr

$$
\sigma_{n}\left(t_{n}\right) \rightarrow 0 \quad \text { as } t_{n} \rightarrow 0 .
$$

Also by Lemma 3

$$
\sum_{1}^{n} \nu b_{\nu}=o(n)
$$


and Lemma 4 now yields (3.3). Finally from (2.5) with $c_{n}=b_{n} \sin n t$, applying (3.4) and Lemma 2,

$$
\left|s_{n}(t)\right|<\epsilon+\frac{2 n \epsilon}{\nu+1}+\sum_{n+1}^{n+\nu}\left|b_{k}\right|, \quad \text { for }|t|<\delta(\epsilon) \text { and } n>n_{0}(\epsilon) .
$$

Write

then by (3.3)

$$
\lim \sup \sum_{n}^{\lambda n}\left|b_{\nu}\right|=v(\lambda)
$$

$$
v(\lambda) \rightarrow 0 \text { as } \lambda \downarrow 1 .
$$

We now choose $\nu=\left[n \epsilon^{1 / 2}\right]$, then, as in $\$ 2$,

$$
\left|s_{n}(t)\right|<3 \epsilon^{1 / 2}+2 v\left(1+\epsilon^{1 / 2}\right) \quad \text { for }|t|<\delta(\epsilon) \text { and } n>n_{1}(\epsilon) \text {, }
$$

which proves the theorem.

COROLLARY. Under the assumptions of Theorem 2

$$
n^{-1} \sum_{1}^{n} \nu b_{\nu} \sin \nu t \rightarrow 0 \text { uniformly at } t=0 .
$$

This follows from $s_{n}(t)-\sigma_{n}(t)=(n+1)^{-1} \sum_{1}^{n} \nu b_{\nu} \sin \nu t$.

4. A converse theorem. To prove a converse of Theorem 2, we introduce the lemma.

LEMMA 5. Suppose that $B_{n} \geqq 0$, that for some $c>0$

$$
B_{n+1} \leqq(1+c / n) B_{n}, \quad n=1,2,3, \cdots,
$$

and that the sequence $\left\{B_{n}\right\}$ is Abel summable to $B$; then $B_{n} \rightarrow B$.

It is known that $B_{n} \geqq 0$ and Abel summability imply $(C, 1) B_{n} \rightarrow B$, that is

$$
B_{n}^{\prime}=\sum_{1}^{n} B_{\nu} \sim n B
$$

From (4.1)

hence

$$
B_{n+k} \leqq(1+c / n)^{k} B_{n}, \quad k=0,1,2, \cdots,
$$

$$
\sum_{k=0}^{\nu} B_{n+k} \leqq B_{n} \sum_{k=0}^{\nu}(1+c / n)^{k}=n B_{n} c^{-1}\left\{(1+c / n)^{\nu+1}-1\right\},
$$

or $B_{n} \geqq c n^{-1}\left\{(1+c / n)^{\nu+1}-1\right\}^{-1}\left(B_{n+\nu}^{\prime}-B_{n-1}^{\prime}\right)$. To any given $\delta>0$ choose $\nu=[\delta n]$, so that $\nu n^{-1} \rightarrow \delta$. Then 
$\liminf _{n \rightarrow \infty} B_{n} \geqq c\left(e^{c \delta}-1\right)^{-1}\{(1+\delta) B-B\}=c \delta B\left(e^{c \delta}-1\right)^{-1} ;$

letting $\delta \downarrow 0$, we get

$$
\lim \inf B_{n} \geqq B .
$$

Similarly from (4.1) by induction

$$
B_{n-k} \geqq(1+c /(n-k))^{-k-1} B_{n+1} \geqq(1+c / \nu)^{-k-1} B_{n+1}
$$

hence

$$
\text { for } n-k \geqq \nu>0 \text {, }
$$

$$
\sum_{k=0}^{n-\nu} B_{n-k} \geqq B_{n+1} \sum_{k=0}^{n-\nu}\left(1+\frac{c}{\nu}\right)^{-k-1}=\nu B_{n+1} c^{-1}\left\{1-\left(1+\frac{c}{\nu}\right)^{\nu-n-1}\right\},
$$

or

$$
B_{n+1} \leqq c \nu^{-1}\left\{1-(1+c / \nu)^{\nu-n-1}\right\}^{-1}\left(B_{n}^{\prime}-B_{\nu-1}^{\prime}\right) \text {. }
$$

Again to any given positive $\delta<1$ choose $\nu=[n \delta]$; then

$\lim \sup B_{n+1} \leqq c\left\{1-e^{c-c \delta^{-1}}\right\}^{-1}\left(B \delta^{-1}-B\right)=c \delta^{-1} B \frac{1-\delta}{1-\exp \left(c-c \delta^{-1}\right)}$.

Letting $\delta \uparrow 1$ we find

$$
\lim \sup B_{n} \leqq B
$$

(4.3) and (4.4) prove the lemma.

It is easily seen that the assumption (4.1) is equivalent to saying $n^{-\gamma} B_{n}$ is decreasing for some $\gamma$; our lemma is in close connection to a lemma due to Hardy [3, p. 442].

Theorem 3. Suppose that $\psi(t) \sim \sum b_{n} \sin n t$, that

$$
\psi(t) \rightarrow \pi A / 2
$$

as $t \downarrow 0$,

and that for some constants $p$ and $c$

$$
0 \leqq(n+1) b_{n+1}+p \leqq(1+c / n)\left(n b_{n}+p\right), \quad n \geqq 1 .
$$

Then $n b_{n} \rightarrow A$.

Let

and

$$
g(t)=(\pi-t) / 2=\sum_{1}^{\infty} n^{-1} \sin n t, \quad 0<t \leqq \pi
$$

(4.7) $\chi(t)=\psi(t)-A g(t) \sim \sum\left(b_{n}-A_{n}{ }^{-1}\right) \sin n t \equiv \sum \beta_{n} \sin n t$, 
then, from (4.5),

$$
\chi(t) \rightarrow 0
$$

Furthermore

$$
n \beta_{n}=n b_{n}-A \geqq-p-A=-q,
$$

say, and

$$
(n+1) \beta_{n+1}+q=(n+1) b_{n+1}+p \leqq(1+c / n)\left(n \beta_{n}+q\right) .
$$

Thus we need only prove $n \beta_{n} \rightarrow 0$, that is Theorem 3 is reduced to the case $A=0$. Now for this case Theorem 2 yields $\sum_{1}^{n} \nu b_{\nu}=o(n)$; finally Lemma 5 applied to $B_{n}=n b_{n}+p$ gives Theorem 3 .

$A$ special case. Let $p=0 ; c=1$; then (4.6) reduces to $0 \leqq b_{n+1} \leqq b_{n}$.

For this case and $A=0$ the theorem is due to Chaundy and Jolliffe, while for $A \neq 0$ it is due to Hardy $[3,4]$. As Hardy remarked, here the case $A \neq 0$ is not immediately reducible to the case $A=0$. Our generalization has the advantage of such reduction.

5. On Gibbs' phenomenon. We shall apply Theorem 2 to the Gibbs' phenomenon (cf. [7, p. 181]). Consider again the assumption (4.5); that is $\psi(t)$ has the jump $\pi A$, while $\chi(t)$ is continuous at $t=0$. We assume in addition (3.2); then evidently the $\beta_{n}$ satisfy the same assumption, hence by Theorem 2

$$
\sum_{1}^{n} \nu \beta_{\nu}=\sum_{1}^{n} \nu b_{\nu}-n A=o(n),
$$

and the series (4.7) is uniformly convergent at $t=0$. On the other hand Fejér proved that

$$
\underset{t_{n} \downarrow 0}{\lim \sup _{1}} \sum_{1}^{n} \nu^{-1} \sin \nu t_{n}=\lim _{n t_{n} \rightarrow \pi} \sum_{1}^{n} \nu^{-1} \sin \nu t_{n}=\int_{0}^{\pi} t^{-1} \sin t d t,
$$

hence assuming, as we may, $A>0$,

(5.1) $\quad \lim \sup _{t_{n} \downarrow 0} \sum_{1}^{n} b_{\nu} \sin \nu t_{n}=\lim _{n t_{n} \rightarrow \pi} \sum_{1}^{n} b_{\nu} \sin \nu t_{n}=A \int_{0}^{\pi} t^{-1} \sin t d t$.

We have thus proved the theorem:

THEOREM 4. Suppose that $\psi(t) \sim \sum b_{n}$ sin $n t$ satisfies the conditions (4.5) and (3.2); then

and

$$
\sum_{1}^{n} \nu b_{\nu} \sim A n
$$




$$
\sum_{1}^{n} b_{\nu} \sin \nu t_{n}-A \sum_{1}^{n} \nu^{-1} \sin \nu t_{n} \rightarrow 0 \quad \text { as } t_{n} \rightarrow 0 ;
$$

in particular (5.1) holds, that is the two series of $\psi(t)$ and $A g(t)$ present the same phenomenon of Gibbs.

For the special case $n b_{n}=O(1)$ Gibbs' phenomenon was observed by Rogosinski [5, pp. 134-135], however it is difficult to follow his argument.

6. A contre example. We cannot replace in Theorems 1 and 2 the conditions (1.6) and (3.2) by (1.3) with $s_{n}=\sum_{1}^{n} a_{\nu}$ or $s_{n}=\sum_{1}^{n} b_{\nu}$ respectively. This is seen from an example constructed by Fejér [2] for a similar purpose. It is a power series $\sum_{k=1}^{\infty} c_{k} z^{k}$ with the following properties [2, pp. 38-46]: The coefficients are all real; the power series is convergent for $|z| \leqq 1$; the function $f(z)=\sum c_{k} z^{k}$ is continuous for $|z| \leqq 1$; the power series is uniformly convergent for $z=e^{i t}, \epsilon \leqq t \leqq 2 \pi$ $-\epsilon, \epsilon>0$, but neither of the series $\sum a_{k} \cos k t, \sum a_{k} \sin k t$ is uniformly convergent for $|t| \leqq \epsilon$. It follows easily that neither series is uniformly convergent at $t=0$, for this would imply uniform convergence on the entire unit circle.

\section{LITERATURE}

1. L. Fejér, Über die Bestimmung des Sprunges der Funktion aus ihrer Fourierreihe, J. Reine Angew. Math. vol. 142 (1913) pp. 165-168.

2. - - Über Potenzreihen, deren Summe im abgeschlossenen Konvergenzkreise iberallstetig ist, Sitzungsberichte der K. Akademie der Wissenschaften, Munich, 1917, pp. 33-50.

3. G. H. Hardy, Some theorems concerning trigonometrical series of a special type, Proc. London Math. Soc. (2) vol. 32 (1930) pp. 441-448.

4. G. H. Hardy and W. W. Rogosinski, On sine series with positive coefficients, J. London Math. Soc. vol. 18 (1943) pp. 50-57.

5. W. Rogosinski, Abschnittsverhalten bei trigonometrischen und insbesondere Fourierschen Reihen, Math. Zeit. vol. 41 (1936) pp. 75-136.

6. O. Szász, Convergence properties of Fourier series, Trans. Amer. Math. Soc. vol. 37 (1935) pp. 483-500.

7. A. Zygmund, Trigonometrical series, Lwow, 1935.

University of Cincinnati 